\title{
Psoriasis and Diabetes, a Dangerous Association: Evaluation of Insulin Resistance, Lipid Abnormalities, and Cardiovascular Risk Biomarkers
}

\author{
Valeria Brazzelli ${ }^{1}$, Pamela Maffioli ${ }^{2,3}$, Vittorio Bolcato ${ }^{4}$, Christian Ciolfi ${ }^{1}$, \\ Angela D'Angelo ${ }^{2,5}$, Carmine Tinelli ${ }^{6}$ and Giuseppe Derosa ${ }^{2,3,5 *}$ \\ ${ }^{1}$ Institute of Dermatology, Fondazione Istituto di Ricovero e Cura a Carattere Scientifico Policlinico San Matteo, University of \\ Pavia, Pavia, Italy, ${ }^{2}$ Department of Internal Medicine and Therapeutics, Centre of Diabetes and Metabolic Diseases, \\ University of Pavia and Fondazione Istituto di Ricovero e Cura a Carattere Scientifico Policlinico San Matteo, Pavia, Italy, \\ ${ }^{3}$ Centre for Prevention, Surveillance, Diagnosis and Treatment of Rare Diseases, Fondazione Istituto di Ricovero e Cura a \\ Carattere Scientifico Policlinico San Matteo, Pavia, Italy, ${ }^{4}$ Department of Public Health, Experimental and Forensic Medicine, \\ Forensic Science Section, University of Pavia, Pavia, Italy, ${ }^{5}$ Laboratory of Molecular Medicine, University of Pavia, Pavia, Italy, \\ ${ }^{6}$ Clinical Epidemiology and Biometric Unit, Fondazione Istituto di Ricovero e Cura a Carattere Scientifico Policlinico San \\ Matteo, Pavia, Italy
}

OPEN ACCESS

Edited by:

Diamant Thaci,

University of Lübeck, Germany

Reviewed by:

Khalaf Kridin,

Rambam Health Care Campus, Israel

Elisabeth Ammitzboll,

Private Practitioner, Hareskov

Hudklinik, Værløse, Denmark

*Correspondence:

Giuseppe Derosa

giuseppe.derosa@unipv.it

Specialty section:

This article was submitted to

Dermatology,

a section of the journal

Frontiers in Medicine

Received: 12 September 2020 Accepted: 15 January 2021 Published: 23 March 2021

Citation:

Brazzelli V, Maffioli P, Bolcato V, Ciolfi C, D'Angelo A, Tinelli C and

Derosa G (2021) Psoriasis and Diabetes, a Dangerous Association: Evaluation of Insulin Resistance, Lipid Abnormalities, and Cardiovascular

Risk Biomarkers.

Front. Med. 8:605691. doi: 10.3389/fmed.2021.605691
Aims: Psoriasis is an immune-mediated dermatosis with cardio-metabolic comorbidities. The aim of this study was to assess insulin-resistance, lipid abnormalities, and cardiovascular risk biomarkers in psoriatic patients with or without type 2 diabetes mellitus (T2DM).

Methods and materials: We enrolled 425 patients: 86 psoriatics, 69 psoriatics with T2DM, 120 T2DM patients, and 150 healthy subjects. We measured the Psoriasis Area and Severity Index (PASI), body mass index (BMI), insulin-resistance parameters [glycosylated hemoglobin $\left(\mathrm{HbA}_{1 \mathrm{c}}\right)$, fasting plasma glucose (FPG), fasting plasma insulin (FPI), and with homeostasis model assessment index (HOMA index)], lipidic panel, plasminogen activator inhibitor-1 (PAI-1), homocysteine, soluble adhesion molecules, matrix metalloproteinase, and adipocytokines.

Results: FPG, $\mathrm{HbA}_{1 \mathrm{c}}$, and $\mathrm{HOMA}-\mathrm{IR}$ were higher in diabetics with psoriasis $(p<0.0001)$ than in psoriatics. FPI levels were higher in diabetics with psoriasis than in diabetics and psoriatics $(p<0.0001)$, and higher in psoriatics than controls $(p<0.0001)$. Psoriatics and diabetics with psoriasis showed higher triglyceride and LDL-C levels $(\rho<0.0001)$ than diabetics. Homocysteine was higher in psoriatics and diabetics with psoriasis $(p<0.0001)$ than in diabetics. PAl-1 was higher in diabetics with psoriasis than diabetics $(p<0.01)$. sICAM-1 and SVCAM-1 were higher in diabetics with psoriasis than diabetics $(p<0.001$ and $p<0.01)$ and psoriatics $(p<0.001$ and $p<0.0001)$. Visfatin and resistin were lower in psoriatics $(p<0.0001)$ and in diabetics with psoriasis $(p<0.001$ and $p<0.0001$, respectively) than diabetics.

Conclusions: A limitation of this study is that there is a significant difference in mean age between controls and other study groups: the lack of matching between case and control groups may interfere with the external validity of the study findings. Despite this, the study 
highlights a pathogenetic link between psoriasis, considered a pre-diabetic condition, and diabetes. Insulin-resistance seems to be the keystone of psoriasis comorbidities. Psoriasis reinforces diabetes, causing a greater cardiometabolic risk.

Keywords: psoriasis, type 2 diabetes mellitus, insulin-resistance, endothelial parameters, obesity

\section{INTRODUCTION}

Psoriasis ( $\mathrm{PsO}$ ) is an immune-mediated inflammatory disease (IMID) affecting the skin and characterized by keratinocyte hyperproliferation and T-lymphocyte inflammation, with a world prevalence of $2-4 \%$ (1). The pro-inflammatory molecules released during chronic inflammation may lead to one or more co-occurring disorders, such as atherogenesis and atherosclerosis, insulin-resistance, hypertension, obesity, dyslipidemia, metabolic syndrome, and type 2 diabetes mellitus (T2DM) (2-4). In literature, patients with psoriasis have an increased risk of new-onset of T2DM (5). Several epidemiological studies reported that the average prevalence of T2DM in psoriatic cohorts was $11.6 \%$ (6). However, other studies that examined the prevalence of T2DM in psoriatic patients compared to controls showed heterogeneous data that do not allow to make a realistic estimate, but sustain an increased prevalence of T2DM in psoriatic cohorts compared to healthy subjects, especially for late onset psoriasis (6). Moreover, a no correlation was observed between T2DM prevalence and psoriasis severity (6).

These two diseases share a common genetic etiology $(7,8)$ and several pathophysiological pathways (9) including those of inflammatory cytokines (10), adipocytokines (10), glucagon-like peptide-1 (GLP-1) receptor, and incretin $(11,12)$.

It was hypothesized that systemic inflammation in psoriasis causes insulin-resistance, an independent T2DM risk factor $(13,14)$. Previous data reported that normal glucose-tolerant patients with moderate to severe psoriasis were more insulinresistant compared to controls suggesting that psoriasis may represent a pre-diabetic condition (15). It was also observed that homeostatic model assessment for insulin-resistance (HOMAIR) values were higher in psoriatic patients compared to control subjects $(16,17)$ and that reduction of the Psoriasis Area and Severity Index (PASI) corresponded to a HOMAIR values decrease (16). Insulin-resistance is also associated with inflammatory cytokines and adipocytokines secreted by adipocytes: levels were comparable between psoriatics and prediabetics (18). Furthermore, it was reported that in psoriatic patients, adiponectin (ADN) and visfatin concentrations were lower, while those of resistin and vaspin were higher with respect to values in control subjects, which suggests that their measurements could be useful to assess the psoriasis disease activity and prevent the onset of its comorbidities (19).

Endothelial damage markers such as soluble adhesion molecules [soluble intercellular adhesion molecule-1 (sICAM1), soluble vascular adhesion molecule-1 (sVCAM-1), soluble E-selectin (sE-selectin)], and metalloproteinases [metalloproteinase-2 (MMP-2) and metalloproteinase-9 (MMP9)] were found elevated in people with $\operatorname{T} 2 \mathrm{DM}(20,21)$ and also involved in psoriasis $(22,23)$. A previous report showed a rise of sICAM-1, sE-selectin, and MMP-9 levels and a trend toward the increase of sVCAM-1 concentrations in psoriatic patients with respect to controls. Since no correlation was found between PASI and these biomarkers, probably they represent an increased systemic rather than skin local inflammation (24).

High levels of plasminogen activator inhibitor-1 (PAI-1), a potent inhibitor of fibrinolysis, have been seen in patients with impaired glucose tolerance (IGT) (25), as well as insulinresistance (26), and could play a role in the pathogenesis of psoriasis. One study showed that in pretreatment, PAI-1 plasma levels were increased in patients with psoriasis compared with healthy subjects, while during treatment they decreased, and this reduction correlated with PASI (27). Furthermore, it was reported that total PAI-1 levels were elevated in psoriatic patients, and they significantly decreased after treatment (24).

The aim of this study was to assess insulin-resistance, lipid abnormalities and cardiovascular risk biomarkers in psoriatic patients with or without T2DM.

\section{METHODS AND MATERIALS}

\section{Study Design}

A case-control study was designed. All study participants provided written informed consent prior to enrollment. Patients were enrolled in the Department of Internal Medicine and Therapeutics, University of Pavia, and in the Institute of Dermatology, Fondazione IRCCS Policlinico, San Matteo, Pavia, Italy. The study protocol was approved by the local Ethical Committee ("Programma Ricerca corrente" clinical code 832rcr2015-1) and registered (ClinicalTrials.gov NCT 02366272).

\section{Study Population}

A total of 155 psoriatic patients $(112 \mathrm{M} / 43 \mathrm{~F})$ were enrolled in the study, subdivided into 86 patients with psoriasis only $(59 \mathrm{M} / 27 \mathrm{~F})$ (mean age $51.46 \pm 15.87)$ and 69 with psoriasis plus T2DM (53M/16F) (mean age $62.67 \pm 8.84)$. Diagnosis of plaque psoriasis was confirmed and assessed using PASI index by a dermatologist. Exclusion criteria for both groups were age $<18$ years old, previous phototherapy, and/or systemic therapy for psoriasis of at least 1-month duration, a diagnosis of erythrodermic or pustular psoriasis, pregnancy or breast-feeding. Furthermore, we also enrolled 120 T2DM (52M/68F) (mean age $60.98 \pm 10.31)$ and 150 healthy subjects as a control population $(82 \mathrm{M} / 68 \mathrm{~F})$ (mean age $33.70 \pm 8.40)$ to undergo the same testing.

\section{Assessments}

Patients underwent initial screening assessment, including medical history, physical examination, evaluation of fasting plasma glucose (FPG), fasting plasma insulin (FPI), glycated hemoglobin $\left(\mathrm{HbA}_{1 \mathrm{c}}\right)$, HOMA-IR, systolic blood pressure 
(SBP), diastolic blood pressure (DBP), total cholesterol (TC), low density lipoprotein-cholesterol (LDL-C), high density lipoprotein-cholesterol (HDL-C), triglycerides (Tg), lipoprotein(a) $[\mathrm{Lp}(\mathrm{a})]$, homocysteine (Hct), PAI-1, sICAM-1, sVCAM-1, sE-selectin, MMP-2, MMP-9, ADN, resistin, visfatin, and vaspin.

All parameters were determined after a $12 \mathrm{~h}$ overnight fast, determined $2 \mathrm{~h}$ after lunch. Venous blood samples were taken for all patients between 8:00 and 9:00 AM and were drawn from an antecubital vein with a 19 -gauge needle, without venous stasis. We used plasma obtained by addition of Na2-EDTA, $1 \mathrm{mg} / \mathrm{ml}$, and centrifuged at $3,000 \times g$ for $15 \mathrm{~min}$ at $4^{\circ} \mathrm{C}$. Immediately after centrifugation, the plasma samples were frozen and stored at $-80^{\circ} \mathrm{C}$ for no more than 3 months. All measurements were performed in a central laboratory.

The Psoriasis Area and Severity Index was evaluated according to Fredriksson and Pettersson's rules (28). Psoriasis was defined as mild if PASI $<10$, moderate if between 10 and 20 , severe if $>20$.

\section{Specific Assessment of Parameters}

For a description of how various parameters were assessed see our previous papers $(21,29)$.

\section{Statistical Analysis}

The Shapiro-Wilk test was used to test the normal distribution of quantitative variables. When quantitative variables were normally distributed, the results were expressed as the mean value and standard deviation (SD), otherwise the median and the interquartile range (IQR; 25-75th percentile) were reported. Qualitative variables were summarized as counts and percentages. One-way ANOVA or Kruskal-Wallis analysis of variance by ranks, as appropriate, with Bonferroni correction, was used to analyze differences among patient groups. Chisquare statistics or Fisher's exact test, as appropriate, was applied to compare qualitative variables. A $p<0.05$ was considered statistically significant. All tests were two-sided. Data analysis was performed using the STATA statistical package (release 15.02018 , Stata Corporation, College Station, Texas, USA).

\section{RESULTS}

\section{Study Sample}

A total of 425 subjects were enrolled in the study (150 healthy controls, 120 diabetics, 86 psoriatics, and 69 diabetics with psoriasis). The characteristics of the study population are described in Table 1.

\section{Body Mass Index}

A significant BMI increase was observed in the studied groups ( $p<0.0001$ for all) compared with (Table 1). Lower BMI was obtained in psoriatics $(p<0.0001)$ and diabetics with psoriasis $(p<0.05)$ compared to diabetics (Table 1). Patients at normal

TABLE 1 | Comparison of quantitative variables among the groups studied.

\begin{tabular}{|c|c|c|c|c|}
\hline & Controls & Diabetics & Psoriatics & Diabetics with psoriasis \\
\hline$N$ & 150 & 120 & 86 & 69 \\
\hline $\operatorname{Sex}(\mathrm{M} / \mathrm{F}) n(\%)$ & 82/68 (54.67/45.33) & $52 / 68(43.33 / 56.67)$ & $59 / 27(68.61 / 31.39)^{\circ \circ^{\star \star \star}}$ & $53 / 16(76.81 / 23.19)^{\star}$ \\
\hline Age (years) & $33.70 \pm 8.40$ & $60.98 \pm 10.31^{0000}$ & $51.46 \pm 15.87^{0000,{ }^{* * \star *}}$ & $62.67 \pm 8.84^{\circ 000, \wedge}$ \\
\hline Duration of DM (years) & $0.00[0.00-0.00]$ & $6.00[3.00-11.00]^{0000}$ & $0.00[0.00-0.00]^{\star \star \star \star}$ & $2.30[1.75-2.60]^{\circ 000, \wedge}$ \\
\hline $\mathrm{BMI}\left(\mathrm{kg} / \mathrm{m}^{2}\right)$ & $23.18 \pm 0.87$ & $30.39 \pm 4.92^{\circ 000}$ & $27.14 \pm 5.93^{\circ 000,,^{\star \star \star \star *}}$ & $29.04 \pm 5.67^{0000, *}$ \\
\hline BMI (18.5-24.9) n (\%) & $150(100)$ & $17(14.17)^{\infty 00}$ & $34(40.00)^{\infty 00,{ }^{* \star \star}}$ & $9(13.04)^{\infty 00, *}$ \\
\hline BMI (25-29.9) n (\%) & $0(0.00)$ & $41(34.17)^{\circ 0 \circ}$ & $32(37.65)^{\infty 00,{ }^{\star \star \star}}$ & $36(52.17)^{\circ 00}$ \\
\hline $\mathrm{BMI}(\geq 30) n(\%)$ & $0(0.00)$ & $62(51.67)^{\circ \infty}$ & $20(23.25)^{\infty 00,{ }^{\star \star \star}}$ & $24(34.78)^{\circ \infty, *}$ \\
\hline $\mathrm{FPG}(\mathrm{mg} / \mathrm{dl})$ & $82.79 \pm 13.29$ & $133.30 \pm 40.45^{\circ 000}$ & $86.39 \pm 8.04^{\star \star \star \star}$ & $161.71 \pm 71.14^{\circ 000_{,}^{\star \star \star \star}, \wedge}$ \\
\hline $\mathrm{FPI}(\mu \mathrm{U} / \mathrm{ml})$ & $6.80[5.20-8.20]$ & $12.35[7.29-20.80]^{0000}$ & $9.70[5.80-16.80]^{0000}$ & $25.80[16.45-57.75]^{0000,{ }^{\star \star \star} \wedge}$ \\
\hline $\mathrm{HbA}_{1 \mathrm{c}}(\%)$ & $4.50 \pm 0.53$ & $6.99 \pm 1.01^{0000}$ & $4.50 \pm 0.53^{\star \star \star \star}$ & $7.41 \pm 1.36^{\circ 000 \wedge}$ \\
\hline HOMA-IR & $1.40 \pm 0.53$ & $4.10 \pm 2.64^{0000}$ & $2.07 \pm 1.28^{\star \star \star \star}$ & $10.26 \pm 6.72^{\circ 000 \wedge}$ \\
\hline $\mathrm{TC}(\mathrm{mg} / \mathrm{dl})$ & $180.85 \pm 25.80$ & $168.31 \pm 38.33^{\circ}$ & $199.27 \pm 34.37^{0000,^{\star \star \star \star \star}}$ & $204.29 \pm 30.6^{\circ 0^{, \star \star \star}}$ \\
\hline LDL-C (mg/dl) & $103.2 \pm 12.7$ & $92.2 \pm 10.8^{\circ}$ & $115 \pm 14.6^{0000, * \star * *}$ & $118.8 \pm 15.9^{\circ 0,,^{* \star \star \star}}$ \\
\hline $\mathrm{HDL}-\mathrm{C}(\mathrm{mg} / \mathrm{dl})$ & $57.81 \pm 12.50$ & $48.98 \pm 14.93^{\circ 000}$ & $58.01 \pm 15.13^{\star \star \star \star}$ & $55.42 \pm 13.11$ \\
\hline $\operatorname{Tg}(\mathrm{mg} / \mathrm{dl})$ & $99.57 \pm 41.70$ & $139.88 \pm 80.56^{\circ 000}$ & $130.69 \pm 71.88^{\circ}$ & $151.75 \pm 70.49^{\circ}$ \\
\hline Lp(a) (mg/dl) & $2.90[2.40-7.20]$ & $18.02[4.32-46.00]^{0000}$ & $12.00[6.00-23.00]^{0000}$ & $7.00[4.50-30.50]^{0000}$ \\
\hline Hct $(\mu \mathrm{mol} / \mathrm{L})$ & $7.74 \pm 2.26$ & $16.86 \pm 6.34^{\circ 000}$ & $23.78 \pm 11.68^{0000,{ }^{, \star \star *}}$ & $24.83 \pm 9.40^{\circ 0000^{, \star \star \star}}$ \\
\hline PAl-1 (ng/ml) & 15.24 [10.96-18.84] & $47.97[27.34-67.00]^{0000}$ & $24.1[12.1-42.9]^{\circ 0,{ }^{* *}}$ & $57.8[20.54-74.96]^{\circ 000,{ }^{\star *}, \wedge}$ \\
\hline
\end{tabular}

Data are represented as $n$ and (\%), means $\pm S D$, median, and interquartile range [IQR].

${ }^{\circ} p<0.05$ vs. controls; ${ }^{\circ} p<0.01$ vs. controls; ${ }^{\circ 00} p<0.001$ vs. controls; ${ }^{\circ 000} p<0.0001$ vs. controls.

${ }^{\star} p<0.05$ vs. diabetics; ${ }^{\star \star} p<0.01$ vs. diabetics; ${ }^{\star \star \star} p<0.001$ vs. diabetics; ${ }^{* \star \star \star} p<0.0001$ vs. diabetics.

${ }^{\wedge} p<0.0001$ vs. psoriatics.

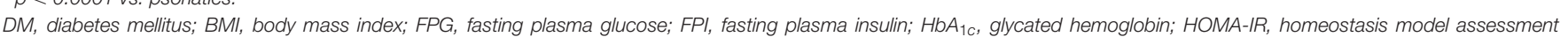

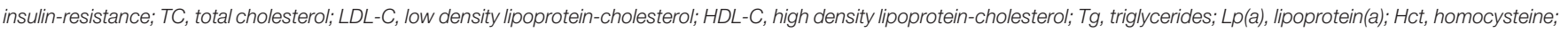
PAl-1, plasminogen activator inhibitor-1. 
weight were $100 \%$ in the control group while psoriatics and diabetics with psoriasis accounted for 14.17, 40.00, and 13.04\%, respectively, of the diabetics group $(p<0.001$ for all vs. controls; $p<0.001$ for psoriatics and $p<0.05$ for diabetics with psoriasis vs. diabetics) (Table 1). Overweight subjects were absent in the control group, whereas they made up $34.17 \%$ of the diabetics, $37.65 \%$ of the psoriatics, and $52.17 \%$ of the diabetics with psoriasis, respectively ( $p<0.001$ for all vs. controls and $p$ $<0.001$ for psoriatics vs. diabetics) (Table 1). Obese subjects were not found among the controls while they made up 51.67, 23.25 , and $34.78 \%$ of the diabetics, psoriatics, and diabetics with psoriasis, respectively, ( $p<0.001$ for all vs. controls; $p<0.001$ for psoriatics, and $p<0.05$ for diabetics with psoriasis vs. diabetics) (Table 1).

\section{Glycemic Control}

A significant FPG, $\mathrm{HbA}_{1 c}, \mathrm{FPI}$, and HOMA-IR increase was observed in diabetics and in diabetics with psoriasis $(p<$ 0.0001 for all) compared to controls (Table 1). FPG, $\mathrm{HbA}_{1 \mathrm{c}}$, and HOMA-IR were found lower in psoriatics $(p<0.0001$ for all) than in diabetics and higher in diabetics with psoriasis $(p<$ 0.0001 for all) respect to psoriatics (Table 1). A significant FPG increase was observed in diabetics with psoriasis $(p<0.0001)$ compared with diabetics, whereas FPI levels were higher in psoriatics $(p<0.0001)$ than in controls and in diabetics with psoriasis compared with diabetics and psoriatics $(p<0.0001$ for all) (Table 1).

\section{Lipid Profile and Lipoprotein Parameters}

Total cholesterol and LDL-C levels were found lower in diabetics $(p<0.05)$ and higher both in psoriatics $(p<0.0001)$ and in diabetics with psoriasis $(p<0.001)$ compared with controls (Table 1). Also, psoriatics and diabetics with psoriasis showed high TC and LDL-C levels $(p<0.0001)$ compared to diabetics (Table 1). A significant decrease of HDL-C concentrations $(p<$ 0.0001 ) was present in diabetics respect to controls whereas an increase was obtained in psoriatics $(p<0.0001)$ compared to diabetics (Table 1). Triglycerides and $\mathrm{Lp}(\mathrm{a})$ levels were higher in diabetics $(p<0.0001$ for both), in psoriatics ( $p<0.01$ and $p<0.0001$, respectively) and in diabetics with psoriasis $(p<$ 0.01 and $p<0.0001$, respectively) compared to controls' baseline value (Table 1).

\section{Fibrinolytic Markers}

A significant PAI-1 and Hct increase was recorded in diabetics ( $p<0.0001$ for both), psoriatics $(p<0.0001$ and $p<0.01$ respectively) and diabetics with psoriasis ( $p<0.0001$ for both) compared with controls (Table 1). Homocysteine levels were higher in psoriatics and diabetics with psoriasis $(p<0.0001$ for both) compared with diabetics (Table 1). A significant decrease in PAI-1 values was observed in psoriatics $(p<0.01)$ compared with diabetics, whereas an increase was observed in diabetics with psoriasis compared with diabetics $(p<0.01)$ and psoriatics $(p<0.0001)$ (Table 1).

\section{Endothelial Parameters}

A significant sICAM-1 and sVCAM-1 increase was observed in diabetics, psoriatics, and diabetics with psoriasis compared with controls ( $p<0.0001$ for both) while sE-selectin levels were higher only in diabetics with and without psoriasis $(p<0.0001)$ than in controls at baseline value (Table 2). A significant increase was obtained for sICAM-1 in diabetics with psoriasis compared with diabetics $(p<0.001)$ and psoriatics $(p<0.001)$ (Table 2). Levels of sVCAM-1 were lower in psoriatics $(p<0.0001)$ than in diabetics whereas they were higher in diabetics with psoriasis compared with diabetics $(p<0.05)$ and psoriatics $(p<0.0001)$ (Table 2). Moreover, a decrease of sE-selectin was obtained in psoriatics $(p<0.0001)$ with respect to diabetics whereas an increase was observed in diabetics with psoriasis $(p<0.0001)$ compared to psoriatics (Table 2).

Matrix metalloproteinase-2 and MMP-9 higher levels were observed in diabetics, psoriatics, and diabetics with psoriasis than in controls ( $p<0.0001$ for both) (Table 2). Moreover, MMP-2 levels were lower only in psoriatics $(p<0.0001)$ compared with diabetics (Table 2). A significant increase was observed for MMP9 in psoriatics and diabetics with psoriasis $(p<0.0001)$ with respect to diabetics and in diabetics with psoriasis $(p<0.0001)$ compared with psoriatics (Table 2).

\section{Adipocytokines}

Adiponectin and visfatin were lower, whereas resistin and vaspin were higher in diabetics, psoriatics and diabetics with psoriasis compared with controls ( $p<0.0001$ for all) (Table 2). A significant reduction was observed for ADN in diabetics with psoriasis compared with diabetics $(p<0.0001)$ and psoriatics $(p<0.05)$ (Table 2). Visfatin and resistin were decreased in psoriatics ( $p<0.0001$ for both) and in diabetics with psoriasis ( $p<0.001$ and $p<0.0001$, respectively) while a significantly reduction of vaspin was observed only in psoriatics $(p<0.001)$ compared with diabetics (Table 2).

\section{PASI Subdivisions}

Patient subdivisions according to PASI index were made, except for control and diabetic patients (Table 3). No significant differences were found among PASI groups ( 0 or absent psoriasis; $<10$, or mild; $10-20$, or moderate; $>20$, or severe) comparing psoriatic patients with patients with diabetes and psoriasis.

\section{DISCUSSION}

Psoriasis is a T cell-mediated inflammatory disease characterized by the expansion and activation of Th-1, Th-17, and Th22 cells, which lead to local overproduction of multiple proinflammatory mediators both by lymphocytes and keratinocytes $(10,30,31)$. Like psoriasis, T2DM is a complex disease with a multifactorial etiology, characterized by peripheral and hepatic insulin-resistance, as well as impaired insulin secretion from pancreatic beta cells. A pathogenetic link between psoriasis and T2DM could be traced primarily in the increase in psoriasis of TNF- $\alpha$, a pro-inflammatory cytokine which plays an important role in the pathogenesis of insulin resistance, through the reduction of the tyrosine-kinase activity of the insulin receptor 
TABLE 2 | Comparison of soluble adhesion molecules, metalloproteinases, and adipocytokines among the groups studied.

\begin{tabular}{|c|c|c|c|c|}
\hline & Controls & Diabetics & Psoriatics & Diabetics with psoriasis \\
\hline$N$ & 150 & 120 & 86 & 69 \\
\hline sICAM-1 (ng/ml) & 202.35 [175.68-248.63] & $245.26\left[_{207.42-301.29]^{+}}\right.$ & 254.64 [202.48-308.62] $^{+}$ & $330.18[273.86-407.66]^{+, \bullet \bullet, \S \S}$ \\
\hline sVCAM-1 (ng/ml) & $449.90 \pm 127.99$ & $627.50 \pm 74.29^{+}$ & $545.13 \pm 138.55^{+, \bullet \bullet \bullet}$ & $719.90 \pm 172.37^{+, \bullet, \S \S \S}$ \\
\hline sE-selectin (ng/ml) & $41.32 \pm 16.02$ & $60.03 \pm 13.27^{+}$ & $40.93 \pm 12.06^{\bullet \bullet \bullet}$ & $57.35 \pm 19.97^{+, \S \S \S}$ \\
\hline MMP-2 (ng/ml) & $453.85 \pm 105.84$ & $752.94 \pm 244.84^{+}$ & $615.40 \pm 242.78^{+, \bullet \bullet \bullet}$ & $638.51 \pm 289.37^{+}$ \\
\hline MMP-9 (ng/ml) & $53.35 \pm 16.24$ & $65.03 \pm 20.41^{+}$ & $75.27 \pm 17.98^{+, \bullet \bullet \bullet}$ & $97.45 \pm 15.17^{+, \bullet \bullet \bullet, \S \S \S ~}$ \\
\hline ADN $(\mu \mathrm{g} / \mathrm{ml})$ & $7.33 \pm 0.45$ & $4.28 \pm 3.31^{+}$ & $3.52 \pm 1.51^{+}$ & $2.09 \pm 0.61^{+, \cdots \bullet »}$ \\
\hline Resistin (ng/ml) & 3.30 [3.10-3.60] & $10.49[9.25-12.59]^{+}$ & $4.82[4.12-5.64]^{+, \bullet \bullet \bullet}$ & $5.10[4.23-5.87]^{+, \bullet \bullet}$ \\
\hline Visfatin (ng/ml) & 17.80 [16.20-23.80] & $8.07[7.30-9.00]^{+}$ & $5.86[4.78-6.89]^{+, \bullet \bullet}$ & $5.88[4.70-7.05]^{+, \bullet \bullet}$ \\
\hline Vaspin (ng/ml) & $0.53[0.46-0.59]$ & $1.54[0.84-2.56]^{+}$ & $0.77[0.57-1.32]^{+, \bullet \bullet \bullet}$ & $0.79[0.62-1.30]^{+}$ \\
\hline
\end{tabular}

Data are means $\pm S D$, median, and interquartile range [IQR].

${ }^{+} p<0.0001$ vs. controls.

$\bullet p<0.01$ vs. diabetics; $\bullet p<0.001$ vs. diabetics; $\cdots p<0.0001$ vs. diabetics.

$\S p<0.05$ vs. psoriatics; $\S^{\S} p<0.001$ vs. psoriatics; $\$ \S \S<0.0001$ vs. psoriatics.

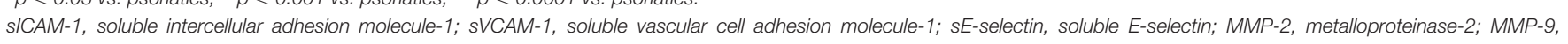
metalloproteinase-9; $A D N$, adiponectin.

TABLE 3 | PASI comparison among the groups studied.

\begin{tabular}{lcc}
\hline & Psoriatics & $\begin{array}{c}\text { Diabetics with } \\
\text { Psoriasis }\end{array}$ \\
\hline$N$ & 86 & 69 \\
PASI $(0=$ absent) & 4 & 0 \\
$n(\%)$ & $(4.71)$ & $(0.00)$ \\
PASI $(<10=$ mild) & 22 & 19 \\
$n(\%)$ & $(25.58)$ & $(27.53)$ \\
PASI (10-20= moderate) & 57 & 46 \\
$n(\%)$ & $(67.06)$ & $(66.66)$ \\
PASI $(>20=$ severe) & 3 & 4 \\
$n(\%)$ & $(3.53)$ & $(5.79)$ \\
\hline
\end{tabular}

Data are expressed as number (n) and percentage (\%).

PASI, Psoriasis Area and Severity Index.

(14). Also, IL-23/Th17 axis was investigated, and its upregulation seems to be central in the explanation of cardiometabolic psoriasis comorbidity $(4,32)$.

As is well-evidenced by Davidovici et al. the production of pro-inflammatory molecules in psoriasis is not limited to the skin, and therefore psoriasis could be considered as a systemic disease $(5,10,33,34)$. The renowned concept of the "psoriatic march" suggests a growing and worsening systemic chronic inflammatory loop with pleiotropic effects on diverse processes, such as angiogenesis, insulin signaling, adipogenesis, lipid metabolism, and immune cell trafficking (33, 35, 36). Therefore, the metabolic aspects of psoriasis have the potential to impact other conditions such as obesity, diabetes, thrombosis, and atherosclerosis $(10,37)$.

Interestingly, our study shows that the levels of FPG, FPI, $\mathrm{HbA}_{1 \mathrm{c}}$, and the HOMA-IR are higher in psoriatics compared with controls, even if only FPI levels resulted statistically significant $(p<0.0001)$, supporting the hypothesis of psoriasis as a "pre-diabetic condition" (15). Moreover, biomarkers of insulin-resistance (FPG and FPI) were especially higher in diabetics with psoriasis compared with diabetics and controls, suggesting also a "positive" reinforcing action of psoriasis on diabetes. The absence of statistically significant differences in PASI subdivisions between psoriatic patients and diabetics with psoriasis, probably excludes similar reinforcing stimulus of diabetes on psoriasis. We could suggest that psoriasis worsens diabetes but that diabetes does not affect psoriasis, if we consider skin lesions. Diabetes, instead, contributes, together with psoriasis, to increase systemic cardiovascular risk. Psoriasis and diabetes together have more harmful action on metabolism than single diseases alone. In view of their lipid profile, psoriatics, and diabetics with psoriasis have TC and LDL-C values higher than controls, but also higher than diabetics. Boehncke et al. supports the synergic effect of the chronic state of inflammation caused by obesity, confirmed by our data where obesity was prevalent in diabetics and psoriatics (1, 10, 14, 38). Among other parameters, adipocytokines secreted by the adipose tissue show numerous functions including the metabolic regulation through lipid and glucose homeostasis. In particular, adiponectin is capable of increasing insulin sensitivity and has a vasoprotective effect acting as antioxidant and anti-inflammatory in the process of early atherogenesis. Our study shows significantly low levels of adiponectin in psoriatics compared to controls and in diabetics with psoriasis compared with diabetics, confirming that the association between psoriasis and diabetes worsens glycemic control and increases cardiovascular risk. Resistin could be considered as an adiponectin "antagonist;" it is higher in diabetics and to a lesser extent in psoriatics, without evidence of further increase in diabetics with psoriasis. Visfatin and vaspin do not yet have precise roles: probably, visfatin has an insulin-resistance activity, while vaspin is an insulin sensitizer. Various studies have reported obesity to be both a cause as well as consequence of psoriasis. In patients with obesity, a dysregulation of adipocytokines is known to occur 
with overproduction of pro-inflammatory adipocytokines such as leptin, visfatin, resistin, and interleukin-6 and decreased production of anti-inflammatory adipocytokines with insulinsensitizing properties such as adiponectin and omentin. The soluble cells' adhesion molecules, such as sICAM-1, sVCAM-1, sE-selectin, and metalloproteinases MMP-2 and MMP-9, given the expression of endothelial dysfunction and plaque alteration, are increased in both diabetics and psoriatics compared with controls, with the exception of sE-selectin increased only in psoriatics compared with controls.

As far as fibrinolytic and vascular markers are concerned, PAI-1 plays a crucial role in the regulation of intravascular fibrinolysis, and its increase is associated with an increase in the risk of occurrence of thrombotic events. Homocysteine is instead a sulfur-containing amino acid, whose increase in the blood is associated with an increased risk of the onset of thrombotic events. Both PAI-1 and Hct are higher in diabetics than in controls and significantly higher in psoriatics than in controls; moreover, in diabetics with psoriasis there are also significantly higher PAI-1 and Hct values than in diabetics, strengthening the contention that psoriasis is associated per se with an increased cardiovascular risk (39-43).

A limitation of this study is that there is a significant difference in mean age between controls and other study groups: the lack of matching between case and control groups may interfere with the external validity of the study findings.

\section{CONCLUSION}

In conclusion, with the limitations described above, this study suggested a close link between psoriasis and diabetes. Insulin resistance seems to be the key to understanding psoriasis comorbidities. Acknowledging psoriasis as a systemic disease, characterized by chronic inflammation, a widespread

\section{REFERENCES}

1. Nestle FO, Kaplan DH, Barker J. Psoriasis. N Engl J Med. (2009) 361:496509. doi: 10.1056/NEJMra0804595

2. Naldi L, Mercuri SR. Epidemiology of comorbidities in psoriasis. Dermatol Ther. (2010) 23:114-8. doi: 10.1111/j.1529-8019.2010.01304.x

3. Mazlin MB, Chang CC, Baba R. Comorbidities associated with psoriasis-data from the malaysian psoriasis registry. Med J Malaysia. (2012) 67:518-21.

4. Gisondi P, Bellinato F, Girolomoni G, Albanesi C. Pathogenesis of chronic plaque psoriasis and its intersection with cardio-metabolic comorbidities. Front Pharmacol. (2020) 11:117. doi: 10.3389/fphar.2020.00117

5. Wan MT, Shin DB, Hubbard RA, Noe MH, Mehta NN, Gelfand JM. Psoriasis and the risk of diabetes: a prospective population-based cohort study. J Am Acad Dermatol. (2018) 78:315-22. doi: 10.1016/j.jaad.2017.10.050

6. Holm JG, Thomsen SF. Type 2 diabetes and psoriasis: links and risks. Psoriasis Targets Ther. (2019) 9:1-6. doi: 10.2147/PTT.S159163

7. Quaranta M, Burden AD, Griffiths CE, Worthington J, Barker JN, Trembath $\mathrm{RC}$, et al. Differential contribution of CDKAL1 variants to psoriasis, Crohn's disease and type II diabetes. Genes Immun. (2009) 10:6548. doi: 10.1038/gene.2009.51

8. Wang H, Wang Z, Rani PL, Fu X, Yu W, Bao F, et al. Identification of PTPN22, ST6GAL1 and JAZF1 as psoriasis risk genes demonstrates shared pathogenesis between psoriasis and diabetes. Exp Dermatol. (2017) 26: 11127. doi: $10.1111 /$ exd.13393 dysimmune background, and dysmetabolic surroundings, allows us to broaden the preventive and therapeutic approach to psoriasis. A healthy lifestyle, prevention and early diagnosis with appropriate therapies can be useful for both pathologies.

\section{DATA AVAILABILITY STATEMENT}

The original contributions presented in the study are included in the article/supplementary materials, further inquiries can be directed to the corresponding author/s.

\section{ETHICS STATEMENT}

The studies involving human participants were reviewed and approved by IRCCS Policlinico San Matteo. The patients/participants provided their written informed consent to participate in this study.

\section{AUTHOR CONTRIBUTIONS}

$\mathrm{GD}, \mathrm{VBr}$, and PM designed the study, researched the data, and wrote the manuscript. CC and VBo researched the data and wrote the manuscript. AD'A processed and analyzed collected samples. All authors contributed to the article and approved the submitted version.

\section{FUNDING}

The research was supported by Fondazione IRCCS Policlinico San Matteo, Pavia, Italy, with a grant for "Ricerca Corrente" project nr. 832-rcr2015-1. The funding source of this study did not have any role in the study design or in conducting the practical work of writing the report.

9. Farh KK, Marson A, Zhu J, Kleinewietfeld M, Housley WJ, Beik S, et al. Genetic and epigenetic fine mapping of causal autoimmune disease variants. Nature. (2015) 518:337-43. doi: 10.1038/nature 13835

10. Davidovici BB, Sattar N, Prinz J, Puig L, Emery P, Barker JN, et al. Psoriasis and systemic inflammatory diseases: potential mechanistic links between skin disease and co-morbid conditions. J Invest Dermatol. (2010) 130:178596. doi: 10.1038/jid.2010.103

11. Faurschou A, Pedersen J, Gyldenlove M, Poulsen SS, Holst JJ, Thyssen $\mathrm{JP}$, et al. Increased expression of glucagon-like peptide-1 receptors in psoriasis plaques. Exp Dermatol. (2013) 22:150-2. doi: 10.1111/exd. 12081

12. Gyldenlove M, Vilsboll T, Zachariae C, Holst JJ, Knop FK, Skov L. Impaired incretin effect is an early sign of glucose dysmetabolism in nondiabetic patients with psoriasis. J Intern Med. (2015) 278:66070. doi: 10.1111/joim. 12388

13. Henseler T, Christophers E. Disease concomitance in psoriasis. J Am Acad Dermatol. (1995) 32:982-6. doi: 10.1016/0190-9622(95)91336-X

14. Boehncke WH. Systemic inflammation and cardiovascular comorbidity in psoriasis patients: causes and consequences. Front Immunol. (2018) 9:579. doi: 10.3389/fimmu.2018.00579

15. Gyldenløve M, Storgaard H, Holst JJ, Vilsbøll T, Knop FK, Skov L. Patients with psoriasis are insulin resistant. J Am Acad Dermatol. (2015) 72:599605. doi: 10.1016/j.jaad.2015.01.004 
16. Karadag AS, Ertugrul DT, Kalkan G, Bilgili SG, Celik HT, Takci Z, et al. The effect of acitretin treatment on insulin resistance, retinol-binding protein4, leptin, and adiponectin in psoriasis vulgaris: a noncontrolled study. Dermatology. (2013) 227:103-8. doi: 10.1159/000351769

17. Ucak S, Ekmekci TR, Basat O, Koslu A, Altuntas Y. Comparison of various insulin sensitivity indices in psoriatic patients and their relationship with type of psoriasis. J Eur Acad Dermatol Venereol. (2006) 20:51722. doi: 10.1111/j.1468-3083.2006.01499.x

18. Rajappa M, Rathika S, Munisamy M, Chandrashekar L, Thappa DM. Effect of treatment with methotrexate and coaltar on adipokine levels and indices of insulin resistance and sensitivity in patients with psoriasis vulgaris. J Eur Acad Dermatol Venereol. (2015) 29:69-76. doi: 10.1111/jdv.12451

19. Coban M, Tasli L, Turgut S, Özkan S, Tunç Ata M, Akin F. Association of adipokines, insulin resistance, hypertension and dyslipidemia in patients with psoriasis vulgaris. Ann Dermatol. (2016) 28:74-9. doi: 10.5021/ad.2016.28.1.74

20. Derosa G, Maffioli P. A review about biomarkers for the investigation of vascular function and impairment in diabetes mellitus. Vasc Health Risk Manag. (2016) 12:415-9. doi: 10.2147/VHRM.S64460

21. Derosa G, D'Angelo A, Tinelli C, Devangelio E, Consoli A, Miccoli R, et al. Evaluation of metalloproteinase 2 and 9 levels and their inhibitors in diabetic and healthy subjects. Diabetes Metab. (2007) 33:12934. doi: 10.1016/j.diabet.2006.11.008

22. Dowlatshahi EA, van der Voort EA, Arends LR, Nijsten T. Markers of systemic inflammation in psoriasis: a systematic review and meta-analysis. $\mathrm{Br}$ J Dermatol. (2013) 169:266-82. doi: 10.1111/bjd.12355

23. Suomela S, Kariniemi AL, Snellman E, Saarialho-Kere U. Metalloelastase (MMP-12) and 92-kDa gelatinase (MMP-9) as well as their inhibitors, TIMP1 and-3, are expressed in psoriatic lesions. Exp Dermatol. (2001) 10:17583. doi: 10.1034/j.1600-0625.2001.010003175.x

24. Sigurdardottir G, Ekman AK, Ståhle M, Bivik C, Enerbäck C. Systemic treatment and narrowband ultraviolet B differentially affect cardiovascular risk markers in psoriasis. J Am Acad Dermatol. (2014) 70:1067-75. doi: 10.1016/j.jaad.2013.12.044

25. Pannacciulli N, De Mitrio V, Marino R, Giorgino R, De Pergola G. Effect of glucose tolerance status on PAI-1 plasma levels in overweight and obese subjects. Obes Res. (2002) 10:717-25. doi: 10.1038/oby.2002.98

26. Declerck PJ, Gils A. Three decades of research on plasminogen activator inhibitor-1: a multifaceted serpin. Semin Thromb Hemost. (2013) 39:35664. doi: 10.1055/s-0033-1334487

27. Nielsen HJ, Christensen IJ, Svendsen MN, Hansen U, Werther K, Brünner $\mathrm{N}$, et al. Elevated plasma levels of vascular endothelial growth factor and plasminogen activator inhibitor-1 decrease during improvement of psoriasis. Inflamm Res. (2002) 51:563-7. doi: 10.1007/PL00012428

28. Fredriksson T, Pettersson U. Severe psoriasis-oral therapy with a new retinoid. Dermatologica. (1978) 157:238-44. doi: 10.1159/000250839

29. Derosa G, Fogari E, D’Angelo A, Bianchi L, Bonaventura A, Romano D, et al. Adipocytokine levels in obese and non-obese subjects: an observational study. Inflammation. (2013) 36:914-20. doi: 10.1007/s10753-01 3-9620-4

30. Boehncke WH. Etiology and pathogenesis of psoriasis. Rheum Dis Clin North Am. (2015) 41:665-75. doi: 10.1016/j.rdc.2015.07.013

31. Di Meglio P, Nestle FO. Immunopathogenesis of psoriasis. In: Clinical and Basic Immunodermatology, 2nd edn. Switzerland: Springer International Publishing (2017). p. 373-95.
32. Egeberg A, Gisondi P, Carrascosa JM, Warren RB, Mrowietz U. The role of the interleukin-23/Th17 pathway in cardiometabolic comorbidity associated with psoriasis. J Eur Acad Dermatol Venereol. (2020) 34:1695706. doi: 10.1111/jdv.16273

33. Armstrong AW, Harskamp CT, Armstrong EJ. Psoriasis and the risk of diabetes mellitus: a systematic review and meta-analysis. JAMA Dermatol. (2013) 149:84-91. doi: 10.1001/2013.jamadermatol.406

34. Billi AC, Gudjonsson JE, Voorhees JJ. Psoriasis: past, present, and future. J Invest Dermatol. (2019) 139:e133-42. doi: 10.1016/j.jid.2019.08.437

35. Brazzelli V, Grasso V, Fornara L, Moggio E, Gamba G, Villani S, et al. Homocysteine, vitamin B12 and folic acid levels in psoriatic patients and correlation with disease severity. Int J Immunopathol Pharmacol. (2010) 23:911-16. doi: 10.1177/039463201002300327

36. Boechat JL. Psoriatic march, skin inflammation and cardiovascular eventstwo plaques for one syndrome. Int J Cardiovasc Sci. (2020) 33:10911. doi: 10.36660/ijcs.20200021

37. Boehncke WH, Boehncke S, Tobin AM, Kirby B. The 'psoriatic march': a concept of how severe psoriasis may drive cardiovascular comorbidity. Exp Dermatol. (2011) 20:303-7. doi: 10.1111/j.1600-0625.2011.01261.x

38. Vanaclocha F, Crespo-Erchiga V, Jiménez-Puya R, Puig L, SánchezCarazo JL, Ferrán M, et al. Immune-mediated inflammatory diseases and other comorbidities in patients with psoriasis: baseline characteristics of patients in the AQUILES study. Actas Dermosifiliogr. (2015) 106:3543. doi: 10.1016/j.ad.2014.06.003

39. Kong Y, Zhang S, Wu R. New insights into different adipokines in linking the pathophysiology of obesity and psoriasis. Lipids Health Dis. (2019) 18:171. doi: 10.1186/s12944-019-1115-3

40. Dattilo G, Imbalzano E, Casale M, Guarneri C, Borgia F, Mondello $\mathrm{S}$, et al. Psoriasis and cardiovascular risk: correlation between psoriasis and cardiovascular functional indices. Angiology. (2018) 69:31-7. doi: 10.1177/0003319717699329

41. Lai YC, Yew YW. Psoriasis as an independent risk factor for cardiovascular disease: an epidemiologic analysis using a national database. J Cutan Med Surg. (2016) 20:327-33. doi: 10.1177/1203475415602842

42. Arican O, Aral M, Sasmaz S, Ciragil P. Serum levels of TNF- $\alpha$, IFN$\gamma$, IL-6, IL-8, IL-12, IL-17, and IL-18 in patients with active psoriasis and correlation with disease severity. Mediators Inflamm. (2005) 2005:2739. doi: 10.1155/MI.2005.273

43. Pereira R, Amladi S, Varthakavi P. A study of the prevalence of diabetes, insulin resistance, lipid abnormalities, and cardiovascular risk factors in patients with chronic plaque psoriasis. Indian J Dermatol. (2011) 56:5206. doi: 10.4103/0019-5154.87144

Conflict of Interest: The authors declare that the research was conducted in the absence of any commercial or financial relationships that could be construed as a potential conflict of interest.

Copyright (C) 2021 Brazzelli, Maffioli, Bolcato, Ciolfi, D’Angelo, Tinelli and Derosa. This is an open-access article distributed under the terms of the Creative Commons Attribution License (CC BY). The use, distribution or reproduction in other forums is permitted, provided the original author(s) and the copyright owner(s) are credited and that the original publication in this journal is cited, in accordance with accepted academic practice. No use, distribution or reproduction is permitted which does not comply with these terms. 